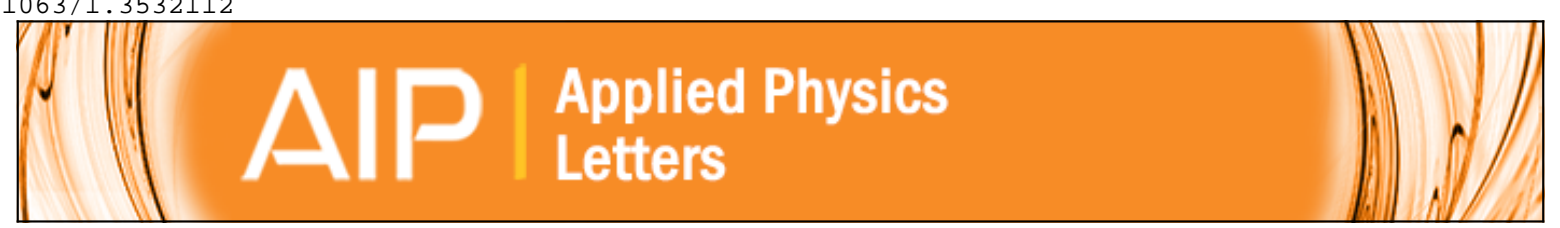

\title{
Characterizing ultrashort optical pulses using second-order nonlinear nanoprobes
}

Haifeng Li, Zhe Zhang, Qian Xu, Kebin Shi, Yaoshun Jia, Baigang Zhang, Yong Xu, and Zhiwen Liu

Citation: Applied Physics Letters 97, 261108 (2010); doi: 10.1063/1.3532112

View online: http://dx.doi.org/10.1063/1.3532112

View Table of Contents: http://scitation.aip.org/content/aip/journal/apl/97/26?ver=pdfcov

Published by the AIP Publishing

Over 700 papers \&
presentations on

multiphysics simulation nww now

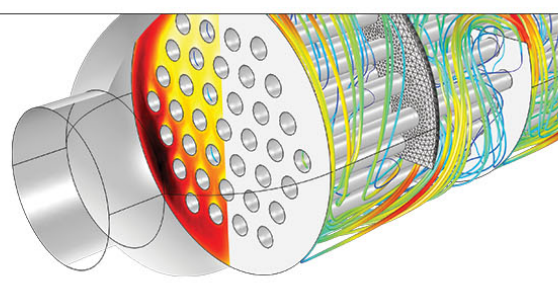




\title{
Characterizing ultrashort optical pulses using second-order nonlinear nanoprobes
}

\author{
Haifeng Li, ${ }^{1}$ Zhe Zhang, ${ }^{1,2}$ Qian Xu, ${ }^{1}$ Kebin Shi, ${ }^{1}$ Yaoshun Jia, ${ }^{3}$ Baigang Zhang, ${ }^{3}$ \\ Yong $\mathrm{Xu}^{3, \mathrm{a})}$ and Zhiwen Liu ${ }^{1, \mathrm{~b})}$ \\ ${ }_{1}^{1}$ Department of Electrical Engineering, The Pennsylvania State University, University Park, \\ Pennsylvania 16802, USA \\ ${ }^{2}$ College of Precision Instrument and Optoelectronics Engineering, Tianjin University, Tianjin 300072, \\ People's Republic of China \\ ${ }^{3}$ Department of Electrical and Computer Engineering, Virginia Tech, Blacksburg, Virginia 24061, USA
}

(Received 11 October 2010; accepted 7 December 2010; published online 29 December 2010)

\begin{abstract}
We report a second-order nonlinear nanoprobe for characterizing ultrafast optical near fields. The proposed nanoprobe comprises second harmonic nanocrystals attached to a carbon nanotube, which is in turn attached to a silica fiber taper. We demonstrate in situ pulse characterization directly in the air core of a photonic crystal fiber. Further, it is shown that nanoprobes containing a single nanocrystal in the tip of the nanotube can be fabricated by auxiliary focused ion beam nanomilling. These results indicate that the proposed nanoprobe can open an avenue for probing the evolution of ultrafast optical fields in complex three-dimensional micro- or nanostructures. (C) 2010 American Institute of Physics. [doi:10.1063/1.3532112]
\end{abstract}

The merging of nanotechnology and ultrafast optics has created opportunities to control photons at nano-femto spatiotemporal scale. ${ }^{1-3}$ In order to advance our capability to engineer ultrafast optical near fields, there is an urgent need to develop techniques to nonperturbingly measure the spatiotemporal evolution of an ultrafast optical near field in nano-femto scale. This is crucial both for coherent control of photons in nano-femto spatiotemporal scale (e.g., to directly measure and hence confirm the engineered nano-femto field) and for elucidating the interaction of ultrafast optical fields and nanoscale systems. Although near-field scanning optical microscopy (NSOM) can achieve nanoscale spatial resolution ${ }^{4}$ and various ultrashort pulse diagnostic tools can characterize femtosecond laser pulses, ${ }^{5,6}$ yet such capability to noninvasively characterize the nanoscale characteristics of femtosecond pulses in all three spatial dimensions remains elusive.

A NSOM fiber probe was utilized to measure the amplitude and the phase of optical fields by heterodyne detection, ${ }^{7}$ visualize the propagation of femtosecond pulses ${ }^{8}$ through photon scanning tunneling microscopy, and characterize ultrashort pulses in the vicinity of the focal point of a lens by interferometry. ${ }^{9}$ Individual second harmonic nanocrystal dispersed on a substrate was also used to characterize focused ultrashort pulses through collinear frequency resolved optical gating (FROG) measurement. ${ }^{10}$ Existing methods, when extended to probing ultrafast optical near field in complex three-dimensional (3D) micro- or nanostructures (e.g., photonic crystal cavities or metamaterials), either require the conversion of local field into propagating waveguide modes (which, as a result, can introduce perturbation) or have limited accessibility due to limited aspect ratio of the probe. Previously, we developed a two-photon fluorescent nanoprobe and demonstrated interferometric autocorrelation

\footnotetext{
a) Tel.: 540-231-2464. Electronic mail: yong@ vt.edu.

b) Author to whom correspondence should be addressed. Tel.: 814-865-2362. Electronic mail: zliu@psu.edu.
}

based pulse characterization. ${ }^{11,12}$ In order to develop the capability to measure both the amplitude and the phase of ultrashort pulses, here we demonstrate a second harmonic nanoprobe (SHARP). The proposed SHARP consists of a silica fiber taper to provide an interface for macroscopic handling and positional control, a single carbon nanotube attached to the taper in order to achieve large aspect ratio, and nanocrystal(s) possessing second-order nonlinearity attached to the nanotube. The nonlinearity of the second harmonic nanocrystal(s) is exploited for femtoscale temporal characterization while nanoscale spatial resolution can be achieved due to the nanoscale dimensions of the nanoprobe. Since SHARP has a large aspect ratio and is built from nanocrystals attached to a nanowire, optical scattering can be minimized and measurements with minimal perturbation can be performed in complex three-dimensional structures.

The fabrication procedure of the proposed SHARP is as follows. A fiber taper with a typical tip diameter of about 1-2 $\mu \mathrm{m}$ was first fabricated from a silica fiber by using a fiber fusion splicer (FSU-975, Ericsson, Brentwood, TN). A single multiwall carbon nanotube (PD100L5-20, NanoLab, Inc., Waltham, MA) was then attached to the taper using an UV curable optical adhesive (Norland optical adhesive 60). The final step involves the attachment of a single or multiple second harmonic nanocrystals to the tip of the carbon nanotube. Barium titanate $\left(\mathrm{BaTiO}_{3}\right)$ nanocrystals (tetragonal phase), which were used for second harmonic imaging probes $^{13}$ and are commercially available (Nanostructured and Amorphous Materials, Inc., Houston, TX), were chosen for this purpose. We dispersed $\mathrm{BaTiO}_{3}$ nanocrystals (suspended in water) on a cover glass (VWR microcover glass) and then let the sample air-dry. By controlling the concentration of the nanocrystals, we were able to attach multiple, a few, or sometimes a single nanocrystal under a home-modified microscope system. As an example, field emission scanning electron microscope images of a SHARP with multiple $\mathrm{BaTiO}_{3}$ nanocrystals are shown in Figs. 1(a) and 1(b). The nanocrystal cluster has a size of about $1-2 \mu \mathrm{m}$ and approxi- 


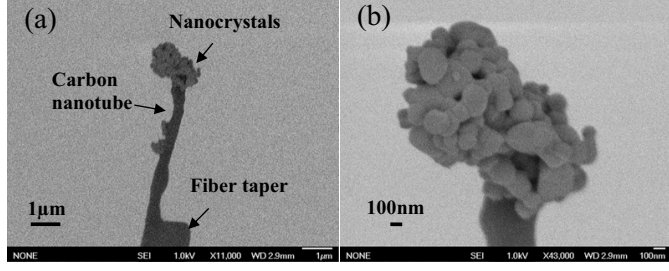

FIG. 1. (a) Field emission scanning electron microscope image of a SHARP; (b) close up view of the attached $\mathrm{BaTiO}_{3}$ nanocrystals.

mately contains 80 nanocrystals (nominal diameter: $200 \mathrm{~nm}$ ). Owing to its small size, phase matching condition is relaxed and therefore our SHARP is also suitable for characterizing ultrashort pulses with a large bandwidth. To ensure that SHARP retains the active nonlinear property of the second harmonic nanocrystal(s), we measured the dependence of second harmonic signal generated from SHARP on the pump power. We found that the second harmonic signal increases nearly quadratically at low pump intensity (e.g., peak power $<$ a few tens $\mathrm{GW} / \mathrm{cm}^{2}$ ). However, at high pump intensity, the signal strength deviates from the quadratic behavior. This phenomenon is currently under investigation and will be reported separately. In the following experiments the pump intensity was kept sufficiently low such that it was within the quadratic response regime.

A unique feature of SHARP is that it is capable of probing an ultrashort optical field in complex micro- or nanostructures due to its high aspect ratio. Here we demonstrate in situ ultrashort pulse characterization directly in the aircore region of a hollow-core photonic crystal fiber. Femtosecond laser beam from a Ti:sapphire mode-locked laser [KMLabs (Boulder, CO), central wavelength: $\sim 754 \mathrm{~nm}$, repetition rate: $\sim 85 \mathrm{MHz}$, and average output power: $\sim 400 \mathrm{~mW}$ ] entered a Michelson interferometer, which was formed by an ultrafast beam-splitter (Newport ultrafast beam-splitter UB.2) and a pair of mirrors. The relative time delay between the resulted two copies of the incoming pulse can be controlled by translating the mirror in one of the arms of the interferometer, which was mounted on a motorized linear translational stage. The two collinearly propagating beams at the output of the interferometer were then focused by an objective $(10 \times$, numerical aperture: 0.25$)$ and coupled into a section of hollow-core photonic crystal fiber (Newport F-AIR-6/800, length: $\sim 15 \mathrm{~cm}$ ), which has an air-core diameter of around $6 \mu \mathrm{m}$ and a zero dispersion wavelength at $753 \mathrm{~nm}$. The femtosecond laser was prechipped using a prism pair, and the center wavelength was tuned to $754 \mathrm{~nm}$ in order to match the minimum dispersion region of the fiber. As illustrated in Fig. 2(a), a SHARP probe can insert into the hollow-core region of the fiber to probe the ultrafast optical near field there in situ. The insets (i) and (ii) show side-view micrographs of a SHARP probe similar to the one shown in Fig. 1 before and after it was inserted into the air core of the fiber. The second harmonic signal generated from the SHARP was then gathered and collimated by a long working distance objective (Mitutoyo 50× M Plan Apo, numerical aperture: 0.55 ) in the forward direction, filtered by a bandpass filter (D400/70, Chroma Technology, Bellows Falls, VT), and finally detected by a spectragraph with a liquid nitrogen cooled charge-coupled device camera (SP2500i, Princeton Acton, Trenton, NJ). The collinear FROG (Refs. 10 and $14-17)$ spectrogram can then be obtained by measur-

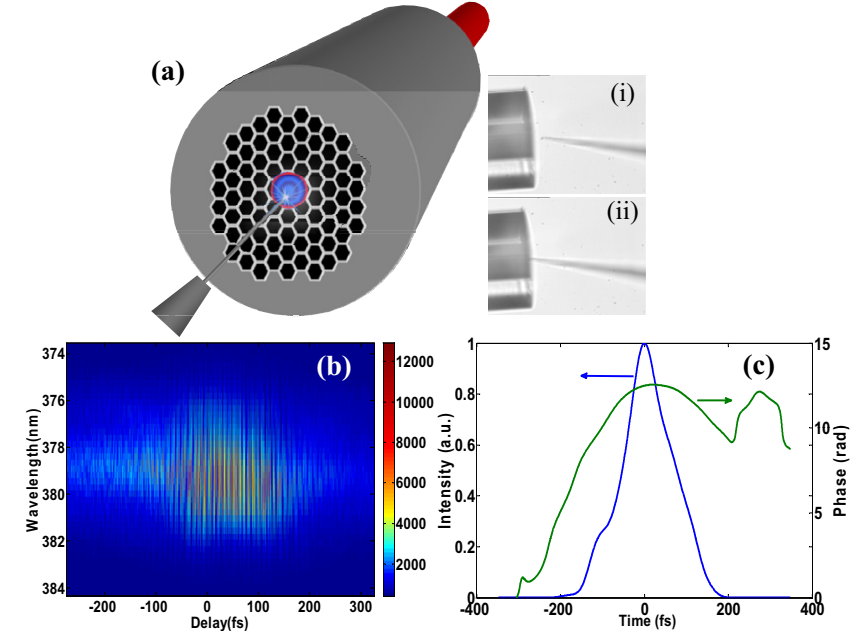

FIG. 2. (Color online) (a) Schematic showing a SHARP is utilized to probe the ultrafast optical near field of a hollow-core photonic crystal fiber; insets (i) and (ii): side-view micrographs of a SHARP before and after it was inserted into a hollow-core photonic crystal fiber. (b) Measured collinear SHG-FROG trace. (c) Retrieved pulse intensity and phase profiles in the air core of a hollow-core photonic crystal fiber.

ing the spectrum of the generated second harmonic signal as the delay time between the two pump beams is scanned. During our experiment, a delay incremental step of $0.67 \mathrm{fs}$ was used. The pump power of each beam measured before the coupling lens (placed in front of the fiber) was around 3 $\mathrm{mW}$. The integration time of the spectragraph was $600 \mathrm{~ms}$. The total elapse time for acquiring a FROG spectrogram was about 15 min. Figure 2(b) shows a measured collinear SHGFROG spectrogram, from which both the amplitude and the phase profile of the ultrashort pulses at the air core can be retrieved. We utilized a method given in Refs. 10 and 16 to retrieve the complex pulse profile. Briefly, we first obtained the usual off-axis second harmonic FROG trace from the collinear FROG spectrogram. The complex pulse profile can then be retrieved using the standard FROG algorithm. ${ }^{5}$ The retrieved pulse intensity and phase profiles are shown in Fig. 2(c). The retrieved pulse width (full width at half maximum) is about $150 \mathrm{fs}$. The pulse is chirped as indicated by a nearly parabolic phase profile. Clearly, our results have shown that SHARP has the unique capability to probe the ultrafast optical near field in complex three-dimensional micro- and nanostructures.

The spatial resolution of SHARP is limited by the size of the active nonlinear component. In order to accomplish true nanoscale resolution, SHARP with a single nanocrystal is required. As aforementioned, by controlling macroscopic parameters such as the concentration of nanocrystals suspended in water, we were able to fabricate SHARPs with the number of nanocrystals ranging from several tens to few and sometimes one. In order to develop a more systematic approach to retain only a single nanocrystal and to precisely define its position relative to the nanotube, we have also exploited focused ion beam (FIB) nanomilling as an additional nanofabrication step, which is capable of both real-time nanoscale imaging and in situ nanomilling. Figure 3 shows two SHARPs before and after FIB nanomilling. A Quanta 200 3D dual beam FIB system was used in this experiment, during which a $\mathrm{Ga}^{+}$ion beam $(20 \mathrm{~nm}, 30 \mathrm{kV})$ was utilized to mill along a line. Both the carbon nanotube and nanocrystals can 


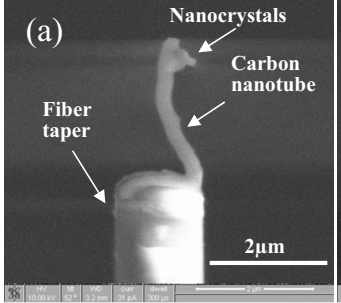

\section{(b)}
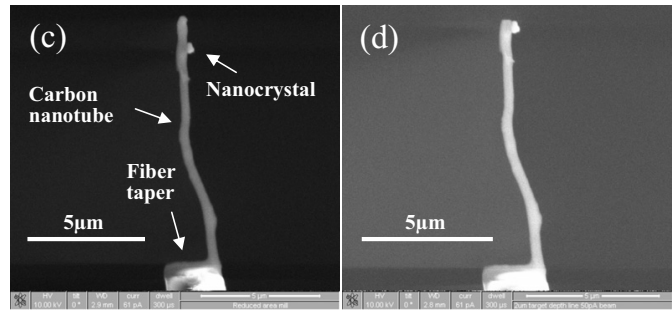

FIG. 3. Fabrication of SHARP by utilizing focused ion beam nanomilling. Two SHARPs [(a) and (c)] before and [(b) and (d)] after FIB nanomilling are shown, respectively. [(a) and (b)] A small section of the carbon nanotube and a protruding nanocrystal were selectively removed by FIB. [(c) and (d)] A small part of the carbon nanotube was removed by FIB to result in a single nanocrystal located at the tip of the nanotube.

be selectively removed. Figures 3(a) and 3(b) show that a small section of the carbon nanotube and a protruding nanocrystal were selectively removed by FIB while in Figs. 3(c) and 3(d) a small part of the carbon nanotube was removed by FIB to result in a single nanocrystal located at the tip of the nanotube.

We utilized the SHARP shown in Fig. 3(d) to probe the pulse profile near the focal point of an objective, as illustrated in Fig. 4(a). The experimental setup is similar except that the output from the Michelson interferometer is focused by an objective $(60 \times$, numerical aperture: 0.85$)$. The pump power of each beam measured before the objective was about $0.2 \mathrm{~mW}$. An integration time of $5 \mathrm{~s}$ was used in our experiment. During the experiment, the center wavelength of the laser was tuned at around $775 \mathrm{~nm}$. The retrieved pulse intensity and phase profiles are shown in Fig. 4(b), which are obtained by using the same retrieval algorithm discussed earlier. A pulse width (full width at half maximum) of 62 fs was
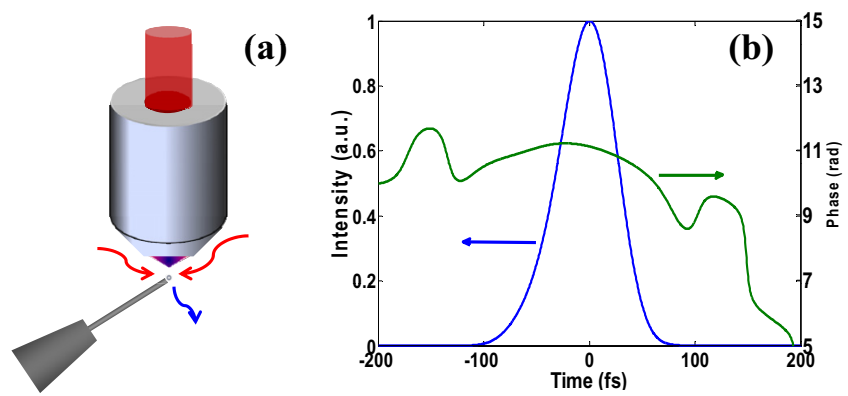

FIG. 4. (Color online) (a) Schematic of in situ characterization of the ultrafast optical field near the focal point of an objective by using a SHARP. (b) Retrieved pulse intensity and phase profiles. obtained. The pulse is slightly chirped. These results have demonstrated the feasibility of fabricating SHARP with a single active nonlinear nanostructure as well as its potential for nano-femto scale spatiotemporal characterization.

In summary, we have proposed and demonstrated a second harmonic nanoprobe or SHARP. The unique fusion of nanoscale and nonlinear features in this device gives rise to its capability to characterize both the amplitude and phase profiles of ultrashort optical pulses potentially with nanoscale spatial resolution. By employing auxiliary FIB nanomilling technique, we show that it is possible to fabricate SHARP containing only a single active nonlinear nanocrystal. We believe that SHARP can provide an avenue toward nano-femto scale spatiotemporal imaging of ultrafast optical field in complex 3D micro- and nanostructures, and is a step forward for fulfilling our dream of having ultimate control of photons and developing capability to engineer arbitrary ultrafast optical near fields.

This work was supported by the National Science Foundation (Award Nos. ECCS0925591, ECCS0644488, DBI0649866, and ECCS0547475). The FIB fabrication was supported by the Pennsylvania State University Materials Research Institute Nanofabrication Laboratory and the National Science Foundation Cooperative Agreement No. 0335765, National Nanotechnology Infrastructure Network, with Cornell University.

${ }^{1}$ M. Aeschlimann, M. Bauer, D. Bayer, T. Brixner, S. Cunovic, F. Dimler, A. Fischer, W. Pfeiffer, M. Rohmer, C. Schneider, F. Steeb, C. Struber, and D. V. Voronine, Proc. Natl. Acad. Sci. U.S.A. 107, 5329 (2010).

${ }^{2}$ M. Durach, A. Rusina, M. I. Stockman, and K. Nelson, Nano Lett. 7, 3145 (2007)

${ }^{3}$ M. I. Stockman, S. V. Faleev, and D. J. Bergman, Phys. Rev. Lett. 88, 067402 (2002).

${ }^{4}$ E. Betzig and J. K. Trautman, Science 257, 189 (1992).

${ }^{5} \mathrm{R}$. Trebino, Frequency-Resolved Optical Gating: The Measurement of Ultrashort Laser Pulses, 1st ed. (Klumer Academic, Boston, MA, 2002).

${ }^{6}$ J.-C. Diels and W. Rudolph, Ultrashort Laser Pulse Phenomena, 2nd ed. (Academic, Boston, 2006).

${ }^{7}$ A. Nesci, R. Dandliker, and H. P. Herzig, Opt. Lett. 26, 208 (2001).

${ }^{8}$ M. L. M. Balistreri, H. Gersen, J. P. Korterik, L. Kuipers, and N. F. van Hulst, Science 294, 1080 (2001).

${ }^{9}$ P. Bowlan, U. Fuchs, R. Trebino, and U. D. Zeitner, Opt. Express 16, 13663 (2008)

${ }^{10}$ J. Extermann, L. Bonacina, F. Courvoisier, D. Kiselev, Y. Mugnier, R. L. Dantec, C. Galez, and J.-P. Wolf, Opt. Express 16, 10405 (2008).

${ }^{11} \mathrm{H}$. Li, Y. Jia, Q. Xu, K. Shi, J. Wu, P. C. Eklund, Y. Xu, and Z. Liu, Appl. Phys. Lett. 96, 021103 (2010).

${ }^{12}$ Y. Jia, H. Li, B. Zhang, X. Wei, Z. Zhang, Z. Liu, and Y. Xu, J. Phys.: Condens. Matter 22, 334218 (2010).

${ }^{13}$ C.-L. Hsieh, R. Grange, Y. Pu, and D. Psaltis, Opt. Express 17, 2880 (2009).

${ }^{14}$ I. Amat-Roldán, I. G. Cormack, P. Loza-Alvarez, and D. Artigas, Opt. Lett. 29, 2282 (2004).

${ }^{15}$ L. Gallmann, G. Steinmeyer, D. H. Sutter, N. Matuschek, and U. Keller, Opt. Lett. 25, 269 (2000).

${ }^{16}$ G. Stibenz and G. Steinmeyer, Opt. Express 13, 2617 (2005).

${ }^{17}$ I. Amat-Roldán, I. Cormack, P. Loza-Alvarez, E. Gualda, and D. Artigas, Opt. Express 12, 1169 (2004). 